\title{
Combination of A Quechers-Based Extraction Protocol with A Fast and Selective UHPLC-QTOF-MS assay For The Detection And Quantification Of Metarhizium Brunneum Metabolites From Honey samples
}

\author{
Judith Taibon ${ }^{1,2}$, Sonja Sturm ${ }^{1}$, Christoph Seger ${ }^{1}$, Hermann Strasser ${ }^{2 *}$ and Hermann Stuppner ${ }^{1}$ \\ ${ }^{1}$ Institute of Pharmacy, Department of Pharmacognosy, CCB - Centrum of Chemistry and Biomedicine, University of Innsbruck, Innrain 80/82, A 6020 \\ Innsbruck, Austria \\ ${ }^{2}$ Institute of Microbiology, University of Innsbruck, Technikerstraße 25, A-6020 Innsbruck, Austria
}

Received: October 4, 2016; Accepted: November 4, 2016; Published: January 2,2017

*Corresponding author: Hermann Strasser, Institute of Microbiology; University of Innsbruck, Technikerstraße 25, A-6020 Innsbruck, Austria; Tel: +43(0)512507/51233; FAX: +43(0)512507/2929; E-Mail: Hermann.strasser@uibk.ac.at

\begin{abstract}
A QuEChERS-based extraction protocol combined with a fast and selective UHPLC-QTOF-MS assay for the detection and quantification of destruxins from honey is described. The internal standard fortified assay showed satisfying assay precision and accuracy and allowed quantification of destruxin A and B down to ppb range with limits of detection (LOD) and quantification (LOQ) of less than $1.0 \mathrm{ppb}$ and 2.3 ppb, respectively. Destruxin E showed a distinctive loss of recovery and was excluded from further quantitative analysis. Honey samples obtained from a field trial using Metarhizium brunneum against the ectoparasitic honey bee mite Varroa destructor were examined for possible destruxin residues. No analytes could be detected and samples were found to be residue-free.
\end{abstract}

Key words: Metarhizium; QuEChER; Honey; Varroa destructor; UHPLC-DAD-QTOF-MS; Risk assessment

\section{Introduction}

Varroa destructor Anderson and Trueman (2000) is an ectoparasitic mite which is currently one of the most serious threats for apiculture $[1,2]$. It was originally confined to the Eastern honey bee Apis cerana however it is nowadays a new parasite of the honey bee Apis mellifer a spread almost worldwide within a short time [3]. Since the honey bee $A$. mellifera is crucial not only for honey production but also for crop pollination varroa control is of great interest [1]. Different approaches to control Varroa destructor have been tried in the last years using mainly synthetic acaricides or organic acids. However, the harmlessness of those substances was questioned the development of alternative treatments is of great necessity $[1,2]$. A promising alternative strategy could therefore be the use of entomopathogenic fungi e.g. Metarhizium spp. or Beauveria $\operatorname{spp}[3,4]$.

Although M. brunneum plays an important role as a biological control agent (BCA) [5] there are still concerns that its bioactive secondary metabolites, so called destruxins (dtxs), imparts risks to humans and the environment. Hence, the availability of analytical assays for destruxin trace analysis is of great necessity in order to answer the question whether destruxins might be able to enter the food chain [6-9].

In this study samples from bee hives treated with M. brunneum strain BIPESCO 5 (listed in regulation EU No. 540/2011 [10]) were examined for possible metabolite residues. Within the following paragraphs the extension of the developed UHPLCQTOF-MS method [7] for the detection and quantification of destruxins from honey samples is presented.

\section{Materials and methods}

\section{Reagents}

Destruxin reference materials (dtxA, dtxB, dtxE, NMR purity greater than 98\%) were isolated from $M$. brunneum strain BIPESCO 5 as previously described [11]. Acetonitrile (gradient grade), was purchased from Merck (Darmstadt, Germany); ammonium formate (for mass spectrometry $\geq 99.0 \%$ ) and roxithromycin (HPLC grade $\geq 90 \%$ ) were purchased from Sigma Aldrich (Vienna, Austria). Nitrogen for mass spectrometry was produced on site by a nitrogen generator (Peak Scientific Instruments, Fountain Crescent, UK). Water for the HPLC was produced on site (Sartorius stedim, arium 611UV, Sartorius, Vienna, Austria).

\section{Preparation of stock solutions and standards}

Always two stock solutions containing either a combination of dtxA, dtxB, and dtxE or the internal standard roxithromycin (IS) (fig. 1) in acetonitrile were prepared. In all cases sample volumes were $1 \mathrm{ml}$. Starting from these stock solutions a series of eight spike solutions containing dtxs and the IS were prepared 


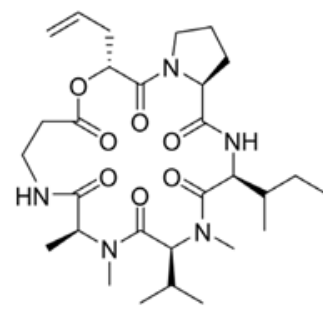

destruxin A

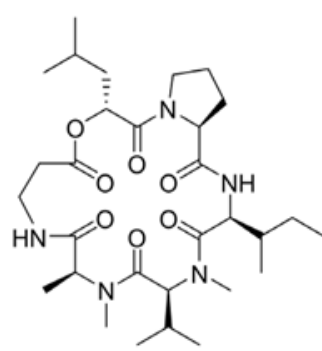

destruxin B

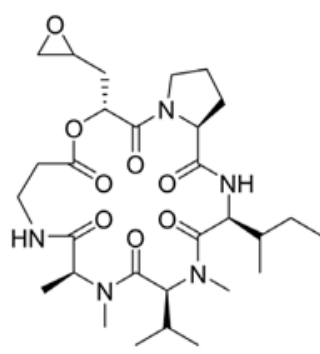

destruxin $\mathrm{E}$

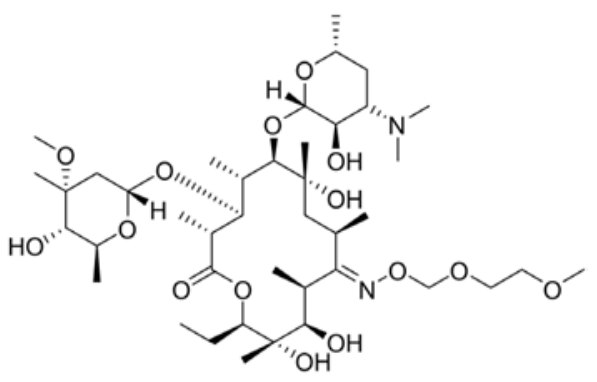

roxithromycin

Figure 1: Structural formulae of the main destruxin congener's dtx A, dtx B, dtx E, and the internal standard roxithromycin.

with following concentrations: $0.05,0.125,0.25,0.375,0.5,1.25$, $2.5,5.0 \mu \mathrm{g} / \mathrm{ml} \mathrm{dtxs}$ and the internal standard at a concentration of $0.5 \mu \mathrm{g} / \mathrm{ml}$. Obtained spike solutions were then used for the preparation of final calibration levels in either acetonitrile by a 1:50 dilution or by spiking $100 \mu \mathrm{l}$ into $5 \mathrm{~g}$ honey sample. A third stock solution containing dtxA, dtxB, dtxE was used to generate quality control samples (QC) for the assessment of precision and accuracy. Spike solutions fortified with different concentrations of the analytes $(0.25,0.5,2.5$ and $3.75 \mu \mathrm{g} / \mathrm{ml})$ and identical amounts of the IS $(0.5 \mu \mathrm{g} / \mathrm{ml})$ were prepared and final QC samples obtained by spiking $100 \mu \mathrm{l}$ into honey sample.

\section{QuEChER-based sample preparation protocol}

Sample preparation was carried out based on the protocol described by Taibon et al. [7]. Samples for calibration curves and quality control samples were spiked with the appropriate amounts of analytes (dtxA, dtxB and dtxE) and the internal standard roxithromycin $(0.5 \mu \mathrm{g} / \mathrm{ml})$, while real samples for destruxin quantification were spiked only with the IS solution $(0.5 \mu \mathrm{g} / \mathrm{ml})$.

Five $g( \pm 0.1 \mathrm{~g})$ of honey samples were weighed into an extraction tube, mixed with $10 \mathrm{ml}$ water and shaken until the honey was completely dissolved. Extraction was carried out with $10 \mathrm{ml}$ of acetonitrile (ACN) followed by dispersive solid phase extraction (d-SPE) for final purification. An aliquot of $1 \mathrm{ml}$ of the purified sample was than evaporated to dryness and re-dissolved in $500 \mu \mathrm{l}$ of acetonitrile prior to analysis.

\section{UHPLC-DAD-QTOF-MS conditions}

An Agilent 1200 UHPLC liquid chromatography (Agilent Technologies, Waldbronn, Germany) equipped with a diode array detector (DAD), an autosampler set at $10^{\circ} \mathrm{C}$, an automatic injector, and a column oven set at $40^{\circ} \mathrm{C}$ was used for all measurements [7]. Separations were performed on a Zorbax Eclipse XDB-C18 rapid resolution column (50 x $2.1 \mathrm{~mm}, 1.8 \mu \mathrm{m}$ particle size, Agilent) guarded with an in-line filter $(0.2 \mu \mathrm{m}$ pore size frit, $2.1 \mathrm{~mm}$ diameter, Agilent) with water (A) and 95\% acetonitrile (v/v) (B), each containing $10 \mathrm{mM}$ ammonium formate, serving as mobile phase. The system was operated at a flow rate of $0.3 \mathrm{ml} / \mathrm{min}$ with following gradient: $\mathrm{t}=0 \mathrm{~min} 75 \% \mathrm{~A}, \mathrm{t}=0.5 \min 75 \% \mathrm{~A}, \mathrm{t}=3.5 \mathrm{~min}$ $60 \% \mathrm{~A}, \mathrm{t}=4.5 \min 50 \% \mathrm{~A}, \mathrm{t}=5.5 \min 35 \% \mathrm{~A}, \mathrm{t}=6.5 \min 5 \% \mathrm{~A}, \mathrm{t}=$ 7.0 min $2 \% \mathrm{~A}, \mathrm{t}=12 \mathrm{~min} 2 \% \mathrm{~A}$. Between runs the column was equilibrated with $75 \%$ A for $12 \mathrm{~min}$. The injection volume was 2.5 $\mu \mathrm{l}$ and chromatograms were recorded at $210 \mathrm{~nm}$.

For destruxin quantification the UHPLC-DAD system was hyphenated to a Bruker micrOTOF-QII mass spectrometer (Bruker Daltonics, Bremen, Germany) operated in positive ESImode. All MS experiments were performed with the ionizer and transfer parameters as described [7].

\section{Assay validation}

Calibration functions were obtained for a dilution series in acetonitrile and in honey serving as matrix model. Spiked matrix samples were prepared using the QuEChERS protocol described above, whereas calibration levels in acetonitrile were directly analysed without further treatment. All calibration levels were measured in triplicates and calibration functions generated using analysis software QuantAnalysis (Version 2.0 SP4, Bruker). For both calibration models the limit of detection (LOD) and the limit of quantification (LOQ) were calculated from calibration functions including only the lowest four fortification levels. From the obtained regression equation data LOD was calculated as three times the standard deviation (STD) of the y-intercept divided by the slope, whereas LOQ was calculated as ten times the STD of the $y$-intercept divided by the slope $[7,12,13]$.

Honey samples, fortified with four different concentrations of analytes and identical amounts of IS, were used to asses precision and accuracy of the assay. For intra- and inter-day repeatability samples were measured on three different days in triplicate. Precision was determined as the relative standard deviation (RSD) of the respective replicate measurements of the different levels; accuracy was expressed as percentage of recovery of the measured analyte concentration in relation to the spiked concentration.

\section{Quantification of destruxins from biological samples}

Honey samples obtained from beehives treated with $M$. 
brunneum (BIPESCO 5) were used for the screening of fungal metabolites [14]. In total 16 beehives, eight for the treatment with BIPESCO5 and eight for control were used. M. brunneum treatment occurred from June to July once a week in four repetitions dusting every frame on both sides with $10 \mathrm{~g}$ of conidial spores per hive (concentration $10^{10} \mathrm{CFU} / \mathrm{g}$ ). For the screening of destruxins honeycombs of every hive filled with honey were taken and stored at $8^{\circ} \mathrm{C}$ until further use.

\section{Results}

\section{Assay validation}

Assay validation was performed with dilution series containing $d t x A, d t x B, d t x E$ and the IS roxithromycin in acetonitrile and honey serving as matrix model. While spiked matrix samples had to be prepared using the described QuEChERS protocol, calibration levels in acetonitrile were directly analysed. Final analyte concentrations in $\mathrm{ng} / \mathrm{ml}$ in acetonitrile corresponded to ppb in matrix samples. All calibration levels were measured in triplicates and results evaluated using the analysis software. Calibration curves were obtained for all three analytes in both matrix models resulting in quadratic functions with correlation coefficient values $\left(\mathrm{R}^{2}\right)$ between 0.9992 and 0.9999 . Calibration ranges for both dilution series in acetonitrile and honey ranged from $1.0-100.0 \mathrm{ng} / \mathrm{ml}$ (ppb) for dtxA and dtxB and from $2.5-$ $100.0 \mathrm{ng} / \mathrm{ml} \mathrm{(ppb)}$ for $\mathrm{dtxE}$. Independent of the matrix model used for calculation limits of detection (LOD) and limits of quantification (LOQ) for dtxs A and B were found to be less than $1 \mathrm{ng} / \mathrm{ml}(\mathrm{ppb})$ and $2.3 \mathrm{ng} / \mathrm{ml}(\mathrm{ppb})$, respectively. Destruxin E showed comparable values for the dilution series in acetonitrile, but much higher results for the dilution series in honey with a LOD of $3.7 \mathrm{ng} / \mathrm{ml}(\mathrm{ppb})$ and a LOQ of $11.3 \mathrm{ng} / \mathrm{ml}(\mathrm{ppb})$. The analytical sensitivity (slope of the calibration function) in reference solutions in acetonitrile was identical to honey matrix samples for dtxA and dtxB but showed a lower value for the calibration curve of dtxE in honey, which indicated a loss of the respective metabolite (tab. 1 ).

Assay precision and accuracy was assessed using spiked matrix samples at four different concentrations each prepared in triplicates. For determination of intra-day and inter-day precision all levels were measured three times on three different days. For all fortification levels of dtxA and dtxB intra-day RSD ranged between $4.9 \%$ and $14.9 \%$ and inter-day RDS was found to be less than $12.6 \%$. Assay accuracy for dtxA and dtxB ranged between $83.4 \%$ and $115.0 \%$ independent on the calibration curve used for calculation (tab. 2). As expected form the differences in the slope of the calibration function for dtxE in acetonitrile and honey matrix, spike experiments confirmed the loss of the respective analyte (Fig. 2). This data are comparable with results obtained in a previously performed study for strawberry and maize proving the assay matrix independent with the exception of dtxE in maize, where also an analyte loss was observed [7]. Consequently quantification of destruxin congeners in honey has to be limited to dtxA and dtxB, which are known as one of the major metabolites secreted by the investigated $M$. brunneum strains $[6,11,13,15,16]$. This allows the use of respective destruxins as surrogate markers for the presence of M. brunneum or its secondary metabolites in honey samples.

\section{Quantification of destruxins from biological samples}

The developed assay was used to screen honey samples obtained from a trial where the fungus Metarhizium brunneum, strain BIPESCO 5, was used to control the ectoparasitic mite Varroa destructor in honey bee colonies. From the original sixteen samples seven controls and seven treatment samples were analysed. All samples were prepared in duplicate and measured three times. No traces of destruxins were found in any of the analysed samples.

\section{Discussion}

With this study the applicability of the developed UHPLCQTOF-MS method has been extended to the analysis of destruxins in honey. The combination of the QuEChERS based sample preparation protocol with the chromatographic system hyphenated to a high resolution TOF mass spectrometer (UHPLCQTOF-MS) allowed quantification of dtxA and dtxB down to ppb range. Overall, the assay showed satisfying sensitivity, precision and accuracy and can be considered as stable and reproducible. The suitability of the assay was further proved by analysing real samples from a field study using M. brunneum to control the ectoparasitic mite Varroa destructor in bee colonies. No traces of destruxins were found in any of the analysed samples.

Monitoring pesticide residues in honey is of great necessity not only for synthetic pesticides but also for biological pest control agents and can help to assess their potential risks to consumer health and environment. Consequently this assay can be considered as a routine method for destruxin trace analysis in honey samples.

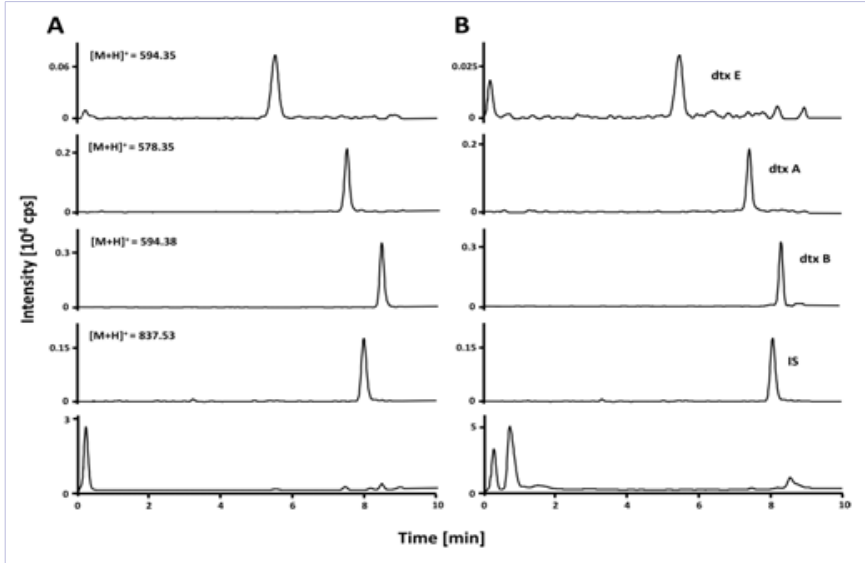

Figure 2: Comparative representation of UHPLC-QTOF-MS ion trace and base peak chromatograms of a spike experiment into acetonitrile (A) and honey (B) matrix. Final concentration of dtx A, B, E and the internal standard roxithromycin were $5 \mathrm{ppb}$ and $10 \mathrm{ppb}$, respectively. 
Table 1: Calibration function parameters for $\mathrm{dtxA}, \mathrm{dtxB}$ and $\mathrm{dtxE}$ diluted in acetonitrile or spiked into honey matrix: calibration range, calibration curve equation, correlation coefficient $\left(R^{2}\right)$, limit of detection (LOD) and limit of quantification (LOQ). All calibration levels were measured three times and calibration curve equation expressed as the mean.

\begin{tabular}{|c|c|c|c|c|c|c|c|c|}
\hline Analyte & Matrix & $\begin{array}{c}\text { Calibration } \\
\text { range }[\mathrm{ng} / \mathrm{ml}]\end{array}$ & $\begin{array}{l}\text { Calibration curve equation }(1 / \mathrm{X}) \\
\qquad y=a x^{2}+b x+c\end{array}$ & $R^{2}$ & $\begin{array}{c}\text { LOD } \\
{[\mathrm{ng} / \mathrm{ml}]^{\mathrm{a}}}\end{array}$ & $\begin{array}{c}\mathrm{LOQ} \\
{[\mathrm{ng} / \mathrm{ml}]^{\mathrm{a}}}\end{array}$ & $\begin{array}{l}\text { LOD in } \\
\text { matrix } \\
\text { [ppb] }^{\text {b }}\end{array}$ & $\begin{array}{l}\text { LOQ in matrix } \\
{[\mathrm{ppb}]^{\mathrm{b}}}\end{array}$ \\
\hline $\operatorname{dtxA}$ & ACN & $1.0-100.0$ & $y=-0.016203 x^{2}+2.987495 x+0.024905$ & 0.9999 & $<1.0$ & 1.7 & & \\
\hline dtxB & ACN & $1.0-100.0$ & $y=-0.042424 x^{2}+4.133664 x+0.151286$ & 0.9999 & $<1.0$ & 2.1 & & \\
\hline dtxE & ACN & $2.5-100.0$ & $y=-0.011095 x^{2}+1.618077 x+-0.043663$ & 0.9999 & $<1.0$ & 2.5 & & \\
\hline $\operatorname{dtxA}$ & honey & $1.0-100.0$ & $y=0.015530 x^{2}+3.231496 x+0.103153$ & 0.9999 & $<1.0$ & 2.2 & $<1.0$ & 2.2 \\
\hline dtxB & honey & $1.0-100.0$ & $y=0.002158 x^{2}+4.536541 x+0.149930$ & 0.9998 & $<1.0$ & 2.3 & $<1.0$ & 2.3 \\
\hline dtxE & honey & $2.5-100.0$ & $y=-0.001306 x^{2}+0.503920 x+0.168720$ & 0.9992 & 3.7 & 11.3 & 3.7 & 11.3 \\
\hline
\end{tabular}

LOD and LOQ were calculated in $\mathrm{ng} / \mathrm{ml}$ for the reference in acetonitrile ${ }^{\mathrm{a}}$ corresponding to $\mathrm{ppb}$ in honey samples ${ }^{\mathrm{b}}$

Table 2: Results for assay accuracy, intra-day and inter-day precision assessed at four different concentration levels using spiked honey samples. Samples of each fortification level were prepared in triplicates and measured three times $(\mathrm{n}=9)$ on three different days. Precision and accuracy was calculated using either the calibration curve in acetonitrile or honey matrix.

\begin{tabular}{|c|c|c|c|c|c|c|c|c|c|c|}
\hline \multirow[b]{2}{*}{ Analyte } & \multirow[b]{2}{*}{ Matrix } & \multirow[b]{2}{*}{ Level $[\mathrm{ng} / \mathrm{ml}]^{\mathrm{a}}$} & \multicolumn{4}{|c|}{ RSD (\%) } & \multicolumn{4}{|c|}{ Accuracy (\%) } \\
\hline & & & day 1 & day 2 & day 3 & day 1-3 & day 1 & day 2 & day 3 & day 1-3 \\
\hline \multirow{4}{*}{$\operatorname{dtxA}$} & \multirow{4}{*}{ ACN } & 5 & 4.9 & 10.9 & 11.2 & 10.2 & 110.9 & 99.1 & 102.1 & 104.0 \\
\hline & & 10 & 6.9 & 9.0 & 9.1 & 9.9 & 114.0 & 101.2 & 115.0 & 110.1 \\
\hline & & 50 & 9.0 & 9.3 & 7.8 & 10.1 & 110.0 & 96.0 & 104.7 & 103.6 \\
\hline & & 75 & 11.9 & 9.3 & 11.3 & 11.4 & 109.0 & 102.3 & 113.6 & 108.3 \\
\hline \multirow{3}{*}{$\operatorname{dtxB}$} & \multirow{3}{*}{$\mathrm{ACN}$} & 5 & 5.4 & 8.3 & 9.1 & 8.3 & 100.9 & 95.5 & 91.9 & 96.1 \\
\hline & & 50 & 8.1 & 10.0 & 9.2 & 10.3 & 113.8 & 102.0 & 101.4 & 105.7 \\
\hline & & 75 & 10.1 & 11.0 & 14.8 & 12.1 & 111.5 & 105.2 & 111.7 & 109.5 \\
\hline \multirow{4}{*}{$\operatorname{dtxA}$} & \multirow{4}{*}{ honey } & 5 & 9.4 & 10.5 & 10.6 & 12.1 & 96.6 & 83.4 & 97.9 & 92.7 \\
\hline & & 10 & 7.2 & 13.1 & 10.9 & 11.0 & 106.4 & 97.7 & 107.0 & 103.7 \\
\hline & & 50 & 14.3 & 9.6 & 13.3 & 12.6 & 101.4 & 94.2 & 100.2 & 98.6 \\
\hline & & 75 & 14.9 & 6.4 & 10.5 & 11.7 & 102.5 & 93.9 & 99.7 & 98.5 \\
\hline \multirow{3}{*}{$\operatorname{dtxB}$} & \multirow{3}{*}{ honey } & 10 & 5.4 & 11.7 & 11.3 & 9.6 & 98.0 & 95.3 & 94.8 & 96.0 \\
\hline & & 50 & 11.9 & 10.2 & 14.2 & 12.0 & 102.7 & 98.5 & 96.4 & 99.2 \\
\hline & & 75 & 13.6 & 7.5 & 12.8 & 11.9 & 102.1 & 93.9 & 95.9 & 97.3 \\
\hline
\end{tabular}

a analyte concentration in the final sample solution corresponds to ppb analytes in honey

\section{Acknowledgement}

This research has been supported by the European Community's Seventh Framework Programme grant (FP7 ENV.2011.3.1.9-1 ECO-INNOVATION, INBIOSOIL, Grant Agreement No. 282767).

\section{References}

1. Kanga LHB, Adamczyk, Patt J, Gracia C, Cascino J. Development of a user-friendly delivery method for the fungus Metarhizium anisopliae to control the ectoparasitic mite Varroa destructor in honey bee, Apis mellifera, colonies. Exp Appl Acarol. 2010;52(4):327-342. doi:10.1007/s10493-010-9369-5.

2. García-Fernández P, Santiago-Álvarez C, Quesada-Moraga E. Pathogenicity and thermal biology of mitosporic fungi as potential microbial control agents of Varroa destructor (Acari: Mesostigmata), an ectoparasitic mite of honey bee, Apis mellifera (Hymenoptera: Apidae). Apidologie. 2008;39(6):662-673. DOI: 10.1051/ apido:2008049.

3. Rosenkranz P, Aumeier P, Ziegelmann B. Biology and control of Varroa destructor. J Invertebr Pathol. 2010; 103:96-119. doi: 10.1016/j.jip

4. Hamiduzzaman MM, Sinia A, Guzman-Novoa E, Goodwin PH.Entomopathogenic fungi as potential biocontrol agents of the ecto-parasitic mite, Varroa destructor, and their effect on the immune response of honey bees (Apis mellifera L.). J Invertebr Pathol. 2012;111(3):237-243.

5. Zimmermann G. Review on safety of the entomopathogenic fungus Metarhizium anisopliae. Biocontrol Sci Technol. 2007;17: 879-920. 
6. Taibon J, Sturm S, Seger C, Parth M, Strasser H, Stuppner H. Development of a fast and selective UHPLC-DAD-QTOF-MS/MS method for the qualitative and quantitative assessment of destruxin profiles. Anal Bioanal Chem. 2014;406(29):7623-7632.

7. Taibon J, Sturm S, Seger C, Strasser H, StuppnerH. Quantitative assessment of destruxins from strawberry and maize in the lower parts per billion range: combination of a QuEChERS-based extraction protocol with a fast and selective UHPLC-QTOF-MS assay. J Agric Food Chem. 2015;63(23):5707-5713. doi: 10.1021/acs.jafc.5b01562.

8. Strauch O, Strasser H, Hauschild R, Ehlers RU.Proposals for Bacterial and Fungal Biocontrol Agents, in: R.-U. Ehlers,editor. Regulation of Biological Control Agents. Springer Netherlands, Dordrecht. 2011;267-288.

9. Strasser H, Hutwimmer S, Burgstaller W. Metabolite Toxicology of Fungal Biocontrol Agents, in: R.-U. Ehlers, editor. Regulation of Biological Control Agents. Springer Netherlands, Dordrecht. 2011;191-213.

10.European Commission, Commission implementing regulation (EU) No 540/2011 of 25 May 2011 implementing Regulation (EC) No $1107 / 2009$ of the European Parliament and of the Council as regards the list of approved active substances, OJEU, L 153/1-186. 2016.

11.Seger C, Eberhart K, Sturm S, Strasser H, Stuppner H. Apolar chromatography on Sephadex LH-20 combined with high-speed counter-current chromatography. High yield strategy for structurally closely related analytes-Destruxin derivatives from Metarhizium anisopliae as a case study. J Chromatogr A. 2006;1117(1):67-73.

12. Harmonized Tripartite Guideline. Validation of Analytical Procedures: Text and Methodology Q2(R1). ICH. 2016.

13. Seger C, Sturm S, Stuppner H, Butt TM, Strasser H. Combination of a new sample preparation strategy with an accelerated highperformance liquid chromatography assay with photodiode array and mass spectrometric detection for the determination of destruxins from Metarhizium anisopliae culture broth. J Chromatogr A. $2004 ; 1061(1): 35-43$.

14. Maira s . Evaluation of a fungal application to increase varroa controlling by Metarhizium anisopliae. Bachelor Thesis. Free University of Bozen. Faculty of Science and Technology. 2013.

15. Loutelier C, Cherton JC, Lange C, Traris M, Vey A. Studies on the dynamics of the production of destruxins by Metarhizium anisopliae. Direct high-performance liquid Chromatographic and fast atom bombardment mass spectrometric analysis correlated with biological activity tests. J Chromatogram A. 1996;738:181-189. doi:10.1016/0021-9673(96)00090-8

16.Wang C, Skrobek A, Butt TM. Investigations on the destruxin production of the entomopathogenic fungus Metarhizium anisopliae. J Invertebr Pathol.2004;85(3):168-174. 